\title{
Application of the Vernier method for absolute distance metrology with CW TOF phase shift technique
}

\author{
Nuno M. Gonçalves ${ }^{1,2, *}$, Manuel Abreu ${ }^{1,2, * *}$, and D. Castro Alves ${ }^{1,2, * * *}$ \\ ${ }^{1}$ Instituto de Astrofísica e Ciências do Espaço, Universidade de Lisboa, Campo Grande, PT1749-016 Lisboa, Portugal \\ ${ }^{2}$ Departamento de Física, Faculdade de Ciências, Universidade de Lisboa, Edifício C8, Campo Grande, PT1749-016 Lisbon, Portugal
}

\begin{abstract}
A phase shift time of flight technique determines a position by comparing the phase angle of a continuously modulated signal in the source and its reflection on a target. However, due to its cyclical properties, the position information is contained within an ambiguity interval. For an absolute measurement, this interval is repeated $N$ times plus a residual part given by the phase shift. In this work we propose an application of the Vernier method to determine $N$ and a setup for mid-range applications (10-20) m with a $3 \mathrm{GHz}$ amplitude modulated source to allow accuracies $\leq 100 \mu \mathrm{m}$.
\end{abstract}

\section{Introduction}

Time of flight (TOF) is a very well established optical measuring technique that offer ways to determine absolute or relative distance measurements (ADM and RDM, respectively). In a phase shift (PS) continuous wave TOF (PS CW TOF) method, a coherent light source is continuously modulated in amplitude and directed to a target. The reflected signal will then have a phase shift, $\Delta \phi$, relative to the original, which will be related to the source-target distance by eq. 1 .

$$
\begin{gathered}
D=\frac{c n}{2 f}\left(\frac{\Delta \phi}{2 \pi}+N\right) \\
\Lambda=\frac{c n}{2 f}
\end{gathered}
$$

Where $f$ is the modulation frequency, $D$ the sourcetarget distance, $c$ the speed of light and $n$ the refractive index of the medium. Due to the cyclical properties of the phase, the position information is contained within an ambiguity distance, eq. 2. Therefore, for an absolute measurement, this interval is repeated $N$ times plus a residual part given by the PS. Hence, it's crucial to determine $N$ for absolute measurements. Different methods can be used for this purpose [1]. Norgia, et al [2] achieved $100 \mu \mathrm{m}$ accuracy for a $2 \mathrm{GHz}$ modulation using heterodyne down conversion (HdC), and solved the ambiguity by using a double Vernier method [3].

For a mid-range (10-20) $\mathrm{m}$, by performing a $3 \mathrm{GHz}$ modulation with $\mathrm{HdC}$, one can achieve an accuracy of $<100 \mu \mathrm{m}$ in an RDM, as long as the error of the frequency and $\mathrm{PS}$ are in the order of $1 \mathrm{kHz}$ and $0.3^{\circ}$, respectively. In this work, we propose a different approach to determine $N$, allowing this level of accuracy for ADM. Although, since

\footnotetext{
*e-mail: fc48080@alunos.fc.ul.pt

**e-mail: maabreu@fc.ul.pt

***e-mail: dmvalves@fc.ul.pt
}

$N$ is an integer, it must be determined with an error $<0.5$. In order to achieve the distance accuracy goal and $N$ being properly determined, a trade off between both of this components must be done. However, the error analysis isn't in the scope of this document.

\section{Working Principle}

In order to measure the source-target distance, the PS CW TOF technique employs the concept of a "ruler". This ruler has a primary and secondary mark, given by the ambiguity distance and by the PS (as it is referred in eq. 1), as shown by ruler $A$ in figure 1 .

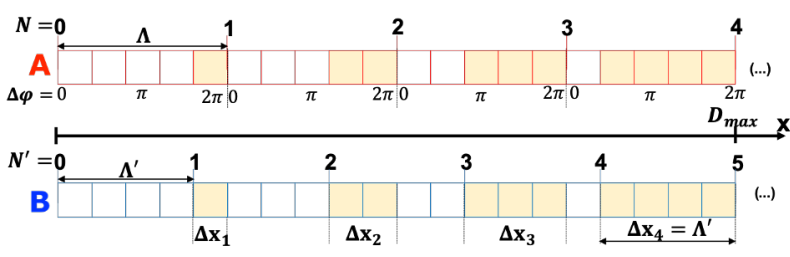

Figure 1. Comparison of two different rulers A and B. In A the distance between marks is determined by $f$ and in $\mathrm{B}$ by $f^{\prime}=$ $f+\Delta f$.

It's possible to determine $N$ by measuring the same distance with two different "rulers". The difference between primary markings of both rulers is defined as $\Delta x_{N}$ :

$$
\Delta x_{N}=N \Delta x_{1} \quad ; \quad \Delta x_{1}=\Lambda-\Lambda^{\prime}=\frac{c n \Delta f}{2 f f^{\prime}}
$$

Where $N$ corresponds to the index of the primary mark of the ruler with biggest spacing between them and $f>$ $\Delta f$ and $\Delta f=f^{\prime}-f>0$ since $f^{\prime}>f$. This represents the difference in frequency for both scales and will rule the variation in position of the different primary marks. 
Additionally, by considering the difference in phase shifts between rulers for the same distance, $\Delta \Phi=\Delta \phi^{\prime}-\Delta \phi$, one gets eq. 4. By rearranging it, the absolute distance can be written as eq. 5 .

$$
\begin{aligned}
& \frac{\Delta \Phi}{2 \pi}=D \frac{2 \Delta f}{c n}+\Delta N \\
& D=\frac{c n}{2 \Delta f}\left(\frac{\Delta \Phi}{2 \pi}-\Delta N\right)
\end{aligned}
$$

Where $\Delta N=N-N^{\prime} \leq 0$ and an integer, since $f^{\prime}>f$ then $\Lambda>\Lambda^{\prime}$ and consequently $N^{\prime} \geq N$. Additionally, by rearranging eq. 1 we obtain eq. 6 . By inserting eq. 5 in eq. 6 and solving for $N$, eq. 7 is obtained.

$$
\begin{gathered}
\frac{\Delta \phi}{2 \pi}=\frac{2 D}{c n} f-N \\
N=\frac{\left(\frac{\Delta \Phi}{2 \pi}-\Delta N\right)}{\Delta f} f-\frac{\Delta \phi}{2 \pi}
\end{gathered}
$$

Considering a target at a fixed distance $D$, one can measure a pair of different phase shifts corresponding to known modulation frequencies spaced by $\Delta f$, by using eq. 7 it's possible to determine the value of $N$.

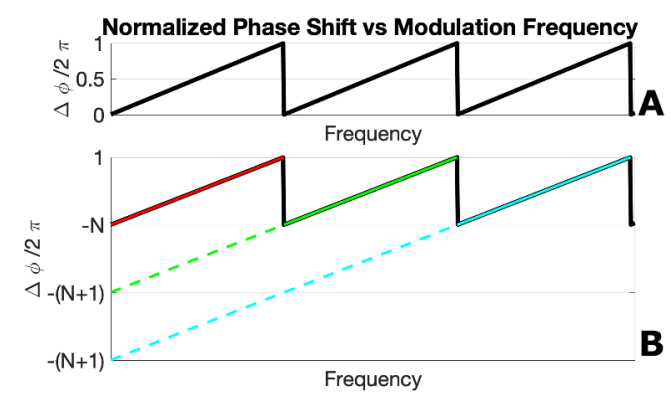

Figure 2. Normalized phase shift for a fixed distance. The colored lines in B are a fit of eq. 6 in the positive slope region of the normalized phase shift.

Very briefly, if one plots eq. 6 for a fixed distance the result is a saw-tooth profile as seen on figure $2 \mathrm{~A}$. For a single and arbitrary frequency-PS point in this profile, the corresponding $N$ can be found as the intercept in the normalized PS axis, as shown in figure 2B. However, for a pair of arbitrary and different frequency-PS points, the $\Delta N$ between them can be $0,-1,-2,-3 \ldots$ depending on the $\Delta f$ spacing. More generally, for any given $D \leq D_{\max }$, by constraining $\Delta f$ one can ensure that for a pair of different frequency-PS points, $\Delta N=0 \bigvee \Delta N=-1$. The maximum distance at which this condition is fulfilled is when $D_{\max }$ is an integer multiple of the ambiguity distances of both frequencies, i.e. the marking of both rulers match and consequently $\Delta \Phi=0$. However, as seen in figure $1, D_{\max }$ will be reached when $\Delta x_{N}=\Lambda^{\prime}$, which mathematically implies that $\Delta N=-1$. By using eq. 5 one can find $D_{\max }$ :

$$
D_{\max }=\frac{c n}{2 \Delta f}(0-(-1))=\frac{c n}{2 \Delta f_{\max }}
$$

Meaning that for $D \leq D_{\max }$ by doing spacings of $\Delta f_{\text {max }}$, as long as $f>\Delta f_{\text {max }}$ for any modulation frequency one can find $\Delta N=0 \bigvee \Delta N=-1$. This is consistent with the results of $S$. Donati [1], that with a different approach showed it was possible to measure unambiguously for $D \leq$ $D_{\max }$.

Additionally, through mathematical analysis of eq. 5, one can conclude that if $\Delta \Phi<0 \rightarrow \Delta N=-1$ and $\Delta \Phi>$ $0 \rightarrow \Delta N=0$. Hence, by measuring a pair of different PS and modulation frequencies spaced at most by $\Delta f_{\text {max }}$ one can know if $\Delta N=0$ or $\Delta N=-1$ if the difference in PS between them is positive or negative.

$$
\text { slope }=\frac{\frac{\Delta \phi^{\prime}-\Delta \phi}{2 \pi}}{\Delta f}=\frac{\Delta \Phi / 2 \pi}{\Delta f}
$$

In conclusion, with eq. 9 and a pair of frequency-PS measurements, one can determine $N$ through eq. 7 . Therefore, by knowing $N$ and applying eq. 1 with a $\mathrm{GHz}$ modulation frequency it's possible to achieve the desired accuracy.

\section{Future work}

In order to show that this principle is valid to obtain absolute distances with high accuracy, we intend to build the setup showed in figure 3. One must ensure that given the instrumental limitation, we can measure for a mid-range distance, $(10-20) \mathrm{m}$, with accuracies $\leq 100 \mu \mathrm{m}$. For that purpose the knowledge of the frequency and PS must be in the order of $1 \mathrm{kHz}$ and $0.3^{\circ}$, respectively. Additionally, since $\mathrm{N}$ is an integer, its measurement error must be smaller than 0.5, to determine the exact integer number of phase cycles to reach the distance D.

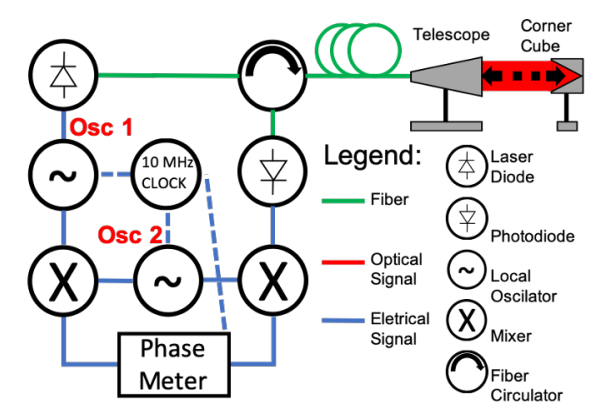

Figure 3. Sensor scheme with $O s c 1$ at $3 \mathrm{GHz}+\Delta f$ and $O s c 2$ at $3 \mathrm{GHz}$.

This work was supported by Fundação para a Ciência e a Tecnologia (FCT) through the research grants UIDB/04434/2020 and UIDP/04434/2020. We also recognize the support of SPOF on the participation of this conference.

\section{References}

[1] S. Donati, Electro-Optical Instrumentation (Pearson Education, New Jersey, 2004) 61-63

[2] M. Norgia, F. Cavedo, A. Pesatori, Proc. IEEE MetroAeroSpace, 408-412 (2017)

[3] M. Rakhmanov, M. Evans, H. Yamamoto, Meas. Sci. Technol. 10, 190-194 (1999) 